\section{P046 GP BASED OXIMETRY AS AN INITIAL DIAGNOSTIC TEST FOR OBSTRUCTIVE SLEEP APNOEA (OSA), OUTCOMES}

Kieran Lee*, Ian Smith. Royal Papworth Hospital, Cambridge Biomedical Campus

\subsection{6/bmjresp-2019-bssconf.46}

Introduction For $>5$ years we have offered a pathway for suspected OSA using a questionnaire and home-oximetry distributed from GP surgeries with interpretation and advice from the sleep centre. We reviewed one year's activity to see if this 'off-tariff' pathway delivers whole-system health economy savings.

Methods Mean ESS and ODI (from home-oximetry) were extracted for referrals on the pathway in 2017-18. Individual charges to the CCG were taken from the hospital finance system. We modelled the cost of home respiratory polygraphy (RP) as an alternative first test, assuming a higher rate of first appointment discharges.

Results We found that 628/5127 (13.3\%) of our new referrals came through the GP pathway. The cost to the CCG of each oximetry alone was $£ 99$. Patients seen in clinic after oximetry were charged as new patients $(£ 276)$ and the oximetry became 'free'. RP was charged at $£ 350$ with a follow up appointment at $£ 110$ and full polysomnography as an inpatient averaged $£ 762$.

228 people (36.3\%) were immediate discharges with advice and no clinic appointment (ODI 4, ESS 9.3); 192 started CPAP, 121 after oximetry (ODI 38, ESS 13.5) and 71 after a further sleep test. All discharged patients were given lifestyle advice and invited to seek re-referral if symptoms persisted/deteriorated. The cost with this system to diagnose a person with OSA needing CPAP was $£ 989$. An initial RP (modelled with 50\% immediate discharges) would have cost $£ 1464$ per CPAP starter. The total saving is estimated at $£ 91281$ p.a. equivalent to $48 \%$ of the current cost.

Conclusion Our OSA pathway reduces travel for patients, as more than a third do not need to attend the hospital. Even with conservative assumptions it offers considerable savings to the local health economy reducing the cost to diagnose a patient with significant OSA by almost a third.

\section{P047 UTILITY OF PEDIATRIC SLEEP QUESTIONNAIRE SLEEP- RELATED BREATHING DISORDER QUESTIONNAIRE SUBSCALE (PSQ-SRBD) IN THE PREDICTION OF OBSTRUCTIVE SLEEP APNOEA (OSA) IN CHILDREN WITH EPILEPSY (CWE)}

\begin{abstract}
1,2Elizabeth Hill", ${ }^{1}$ Laura Hill, ${ }^{1}$ Emma Carruthers, ${ }^{3,4}$ Jay Shetty, ${ }^{3}$ Ailsa McLellan, ${ }^{3,5}$ Richard Chin, ${ }^{5,6}$ Don Urquhart. ${ }^{1}$ Department of Paediatric Cardiac, Respiratory and Sleep Physiology, Royal Hospital for Children and Young People, NHS Lothian, Edinburgh, UKi ${ }^{2}$ Sleep Research Unit, Centre for Clinical Brain Sciences, The University of Edinburgh, Edinburgh, UK; ${ }^{3}$ Department of Paediatric Neurosciences, Royal Hospital for Children and Young People, NHS Lothian, Edinburgh, UK; ${ }^{4}$ Centre for Reproductive Health, The University of Edinburgh, Edinburgh, UK; ${ }^{5}$ Department of Child Life and Health, The University of Edinburgh, Edinburgh, UK; ${ }^{6}$ Department of Paediatric Respiratory and Sleep Medicine, Royal Hospital for Children and Young People, NHS Lothian, Edinburgh, UK
\end{abstract}

\subsection{6/bmjresp-2019-bssconf.47}

Introduction Epilepsy and OSA are common conditions, affecting $0.5 \%{ }^{1}$ and $6 \%{ }^{2}$ of children respectively. Pilot data utilising the PSQ-SRBD reported a high risk of OSA in 55\% of CWE

Abstract P047 Table 1 Summary of anthropometric, questionnaire and polysomnographic data for children with epileptic control children. Groups compared using Student's t-test for continuous variables and chi-square test for discrete variables. Results presented as number(\%), mean \pm SD or median (IQR25-75\%) as appropriate.

\begin{tabular}{|c|c|c|c|}
\hline & & & \\
\hline & $\begin{array}{l}\text { Children with epilepsy } \\
\qquad \mathbf{n}=\mathbf{3 5}\end{array}$ & $\begin{array}{l}\text { Non-epileptic control children } \\
\qquad \mathrm{n}=\mathbf{1 7}\end{array}$ & $\mathbf{p}$ \\
\hline Sex & $\begin{array}{l}21(60 \%) \text { male : } 14(40 \%) \\
\text { female }\end{array}$ & $10(59 \%)$ male : 7 (41\%) female & 1.000 \\
\hline Age (years) & $11 \pm 3$ & $11 \pm 3$ & 0.997 \\
\hline Body mass index (BMl; $\left.\mathrm{kg} / \mathrm{m}^{2}\right)$ & $21.3 \pm 5.4$ & $19.0 \pm 3.8$ & 0.125 \\
\hline Children's Epworth Sleepiness Score (cESS; /24) & $5(3-10)$ & $3(1-5)$ & 0.006 \\
\hline Excessive daytime somnolence (EDS; cESS>10) & $8(23 \%)$ & $0(0 \%)$ & 0.031 \\
\hline PSQ-SRBD score (range 0-1) & $0.35(0.23-0.53)$ & $0.09(0.00-0.25)$ & $<0.0001$ \\
\hline PSQ-SRBD high risk of SRBD (score $\geq 0.33$ ) & $21(60 \%)$ & $2(12 \%)$ & 0.001 \\
\hline & n=29 scored PSGs & n=15 scored PSGs & \\
\hline Obstructive apnoea/hypopnoea index (OAHI; /hour) & $0.1(0.0-0.7)$ & $0.1(0.0-0.4)$ & 0.640 \\
\hline Apnoea/hypopnoea index (AHI; /hour) & $0.5(0.2-1.5)$ & $0.8(0.4-1.0)$ & 0.652 \\
\hline Oxygen desaturation index (ODI; /hr) & $0.3(0.1-1.3)$ & $0.2(0.0-0.7)$ & 0.194 \\
\hline $\mathrm{SpO}_{2}$ nadir (\%) & $93 \pm 3$ & $93.3 \pm 3$ & 0.535 \\
\hline Obstructive sleep apnoea (OSA) positive (oAHI $\geq 1 / \mathrm{hr}$ ) & $5(14 \%)$ & $2(12 \%)$ & 1.000 \\
\hline $\begin{array}{l}\text { Obstructive sleep apnoea/hypopnoea syndrome (OSAHS) positive } \\
\text { (oAHI } \geq 1+\text { ESS }>10)\end{array}$ & $0(0 \%)$ & $0(0 \%)$ & - \\
\hline
\end{tabular}


Abstract P047 Table 2 Utility of PSQI-SRBD questionnaire in predicting obstructive apnoea/hypopnoea index (oAHI) $\geq 1 / \mathrm{hr}$ on polysomnography in children with epilepsy. Results presented as number(\%).

\begin{tabular}{|c|c|c|c|}
\hline & $\mathrm{OAHI} \geq 1 / \mathrm{hr}$ & $\mathrm{OAHI}<1 / \mathrm{hr}$ & \\
\hline PSQ-SRBD $\geq 0.33$ & $3(10 \%)$ & $14(48 \%)$ & 17 \\
\hline \multirow[t]{2}{*}{ PSQ-SRBD $<0.33$} & $2(7 \%)$ & $10(35 \%)$ & 12 \\
\hline & 5 & 24 & \\
\hline
\end{tabular}

Sensitivity $=60 \%$

Specificity $=42 \%$

Positive predictive value $=18 \%$

Negative predictive value $=83 \%$

versus $7 \%$ of controls. ${ }^{3}$ This study assessed symptoms of OSA in CWE and typically-developing children using subjective questionnaires, and compared these with objective level I polysomnography.

Methods Ethical approval was granted. Written informed consent/assent was obtained from participants/caregivers. CWE aged 5-18 years were recruited from an NHS epilepsy clinic during 2017-2019, along with age- and sex-matched typicallydeveloping controls. Children with significant co-morbidities were excluded.

Anthropometric data were collected. OSA symptoms were rated by child/caregiver using the children's Epworth Sleepiness Scale (cESS) and PSQ-SRBD, with PSQ-SRBD $\geq 0.33$ indicative of OSA (reported sensitivity 81\%, specificity $87 \%)^{4}$ Polysomnography (SOMNOScreen plus ${ }^{\mathrm{TM}}$, SOMNOmedics, Randersacker, Germany) was conducted in accordance with AASM Version 2.3 (2016) guidelines. ${ }^{5}$ Standard statistical analyses were undertaken using SPSS 25 (IBM Corp., Armonk, NY, USA). Significance was taken as $\mathrm{p}<0.05$.

Results Fifty-two children completed the protocol (35 CWE, 17 controls), with polysomnography data available for 44 children. Table 1 summarises anthropometric, questionnaire and polysomnography data; CWE were significantly sleepier on cESS and more likely to score $\geq 0.33$ on PSQ-SRBD. Children with PSQ-SRBD $\geq 0.33$ had higher BMI $(22.3 \pm 6.0$ v. 19.1 $\left.\pm 3.7 \mathrm{~kg} / \mathrm{m}^{2}, \quad \mathrm{p}=0.03\right)$, higher cESS (8(4-12) v. 3(1-5), $<0.0001)$ and lower SpO2 nadir on polysomnography $(92 \pm 3$ v. $94 \pm 2, p=0.039)$. The positive predictive value of a score of $\geq 0.33$ on the PSQ-SRBD in CWE was low (18\%), with sensitivity $60 \%$ and specificity $42 \%$ noted in predicting OSA (oAHI >1/hour); see table 2.

Discussion Study limitations include small study numbers and low (o)AHI. Notwithstanding these limitations, CWE had significantly higher cESS and PSQ-SRBD scores than controls, reflecting previously published data. The utility of PSQ-SRBD in predicting OSA in CWE is low, in contrast to studies performed in healthy children. The study is ongoing.

\section{REFERENCES}

1. Joint Epilepsy Council of the UK and Ireland. Epilepsy prevalence, incidence and other statistics 2011.

2. Marcus $\mathrm{CL}$, et al. Diagnosis and management of childhood obstructive sleep apnea syndrome. Pediatrics 2012; 130: e714-e755.
3. Urquhart DS, et al. Observational pilot study of reported symptoms of Obstructive Sleep Apnoea (OSA) in children with epilepsy and healthy controls. Dev Med Child Neurol 2016; 58: 1063-1068.

4. Chervin RD, et al. Pediatric sleep questionnaire (PSQ): Validity and reliability of scales for sleep-disordered breathing, snoring, sleepiness, and behavioral problems. Sleep Med 2000; 1: 21-32.

5. Berry RB, et al. The AASM Manual for the Scoring of Sleep and Associated Events: Rules, Terminology and Technical Specifications, Version 2.3. 2016; Westchester, IL: American Academy of Sleep Medicine.

\section{P048 EVALUATION OF THE UTILITY OF THE EPWORTH SLEEPINESS SCALE (ESS), FOR TRIAGING INSOMNIA REFERRALS FOR ASSESSMENT AND TREATMENT}

Paul Reynolds-Cowie, Peter Venn*, Maja Schaedel, Sue Twyman, Karen Schofield. Queen Victoria Hospital, NHS Foundation Trust, East Grinstead, UK

\subsection{6/bmjresp-2019-bssconf.48}

Introduction Excessive daytime sleepiness is generally less prevalent in insomnia when compared with other sleep disorders. ${ }^{1}$ As a result of this, the Epworth Sleepiness Scale (ESS) ${ }^{2}$ is currently used by our service to triage patients referred by their GP with symptoms of insomnia for assessment. Patients with an ESS 0-3 are triaged straight to assessment in a joint sleep medicine and clinical psychology clinic. Patients with an ESS 4 + are first triaged for further investigation, using actigraphy, oximetry and full overnight polysomnography to look for other elements of sleep disturbance apart from insomnia. This audit aimed to evaluate the effectiveness of the current triaging process and review patient treatment pathways arising from this.

Methods Data was analysed for 131 patients, 86 females (age 16-87, mean 49.6), and 45 males (age 19-84, mean 52.98), from the joint insomnia clinic between February 2018 and March 2019. ESS of 0-3, 4-10, and 11+, were analysed separately to look for associations between the ESS and diagnoses, in order to evaluate the utility of the current triaging process.

Results Figures 1 and 2 show diagnoses/treatment pathways.

As shown as the ESS increased, there was a shift from psychological to physiological sleep pathology. This is also reflected in the change from psychological to medical intervention, with the exception of drug therapy for delayed sleep in ESS 0-3. Those with an Epworth of 4-10 had more of a 